\title{
A NEW METHOD FOR DETERMINATION OF PLASTIC DEFORMATION PROPERTIES AT HIGH STRAIN RATE AND ITS MODELLING
}

\author{
Xiangfan Fang* \\ Institute of Automotive Lightweight Design, University Siegen, D-57076 Siegen, Germany
}

\begin{abstract}
The determination of material properties under high speed loading is a challenge. The system ringing in a conventional servo-hydraulic tensile testing machine deteriorates the quality of force measurement, which makes a precise determination of yield locus, strain hardening and fracture strain difficult. In this work, the system ringing effect of the entire tensile testing system were analyzed. It was determined that the ringing of the system is location and geometry dependent. A new type of tensile sample has been developed. Beside of the usual major plastic deformation area, it has an additional elastic area, within which a locally restricted secondary minor plastic deformation takes place. This very small plastic deformation absorbs the elastic vibrations in this area. Therefore, the deformation forces can be measured by strain gauge without any ringing effect. The plastic deformation behavior of materials can be determined for a wide range of strain rate of $0.0001-1000 / \mathrm{s}$ exactly. To explain the functionality and the physical background of the new sample, based on the equations for onedimensional stress waves and theory of the stress wave attenuation due to dislocation motions, a simplified beam model with analytical formulations could be established und programmed in MATLAB. Verifications show a good prediction of sample geometry using this simplified model.
\end{abstract}

\section{Introduction and state of the art}

During a vehicle crash event, large multi-axial plastic deformation with strain rate in the range of $10^{-4}-10^{3} / \mathrm{s}$ occurs. The material behavior must be exactly determined and modelled for the FE simulation, which is commonly used in the vehicle development process. In order to determine the flow curves in this strain rate range, three different measuring techniques are generally used: quasi-static testing machine with $80 \mathrm{~mm}$ tensile specimen for $10^{-4}-10^{-2} / \mathrm{s}$; servo-hydraulic high-speed testing machine with 20-25 mm smaller specimens according to ISO 26203-2 for the range up to $10^{3} / \mathrm{s}$; Split-Hopkinson-Bar (SHB) for the range $>10^{3} / \mathrm{s}$ with very small 5-10 mm specimens (ISO 26203-1). The experimental techniques and conditions, i.e. the measurement of force, deformation, specimen geometry and size as well as the specimen fixation, are different for the strain rate ranges and produce an apparent discontinuity in material behavior [1].

*Corresponding author: xiangfan.fang@uni-siegen.de 
The use of these different techniques is the results of the force signal oscillations at high strain rates, the so-called system ringing effect $[2,3]$. It is well known, that an impact on a solid body would induce a stress impulse $\sigma$ which is proportional to the loading speed $\mathrm{V}$, the material density $\rho$ and its sound speed $\mathrm{C}$ according to the eqn. 1 [2]:

$$
\sigma=V \cdot \rho \cdot C
$$

Xiao [3] and Hill [4] proposed to use a longer load rising time to solve the system ringing problem caused by the impact stress pulse. The disadvantage is that this pre-condition usually cannot be met due to the short test duration at high-required speeds and the requirement of a certain high strain rate. Other researchers [5-7] reported that forces are measurable with reduced oscillations when strain gauge sensors (SGS) for force measurement are placed on the lower sample shoulder area above the sample clamping area at a strain rate of up to 200 /s. Beyond 200 /s, strong force oscillations are unavoidable, and the common practice is to filter the high-speed tensile force-displacement curves to obtain smooth stress-strain curves.

Using a Split Hopkinson Bar (SHB) the force signals can be measured without any oscillation for the range $>10^{3} / \mathrm{s}$. However, the results of such experiments by different research groups for the same material are considerably different. Verleysen et.al. stated [8] that in a SHB experiment, many of the assumptions are not valid in reality. The stress and strain in the very small specimens are not homogeneous as assumed and the achievement of a quasi-static equilibrium is not always given.

For automotive industry, reliable and low coast standard testing methods are mandatory. Therefore, in this work, the testing method based on servo-hydraulic high-speed tensile machine was analyzed.

\section{Stress wave propagation and ringing free force measurement}

Based on the positive effect determined in [7] for force measurement in the lower area above the sample clamping area of the ISO 26203-2 sample (elastic measurement area [EMA] in Fig. 1a), Li and Fang performed an extensive FE analysis on high-speed tests using a hydraulic machine [9], where the entire hydraulic tensile machine (Zwick Company, type HTM5020) was modeled using LS-DYNA (see also Fig. 1d). Frequency analysis shows that if the eigenfrequency of the sample is higher, the force oscillation in the sample area below the first shoulder (S1) may be further reduced. Therefore, the ISO 26203-2 sample has been modified to the so-called Generation I (Gen. I) sample (Fig. 1b). The force oscillation in the elastic measurement area (EMA) was reduced comparing to the ISO sample.
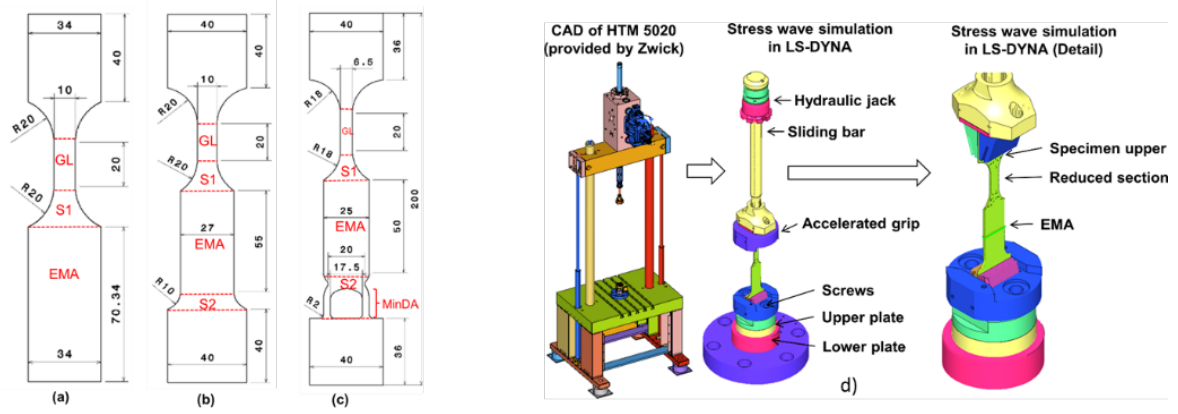

Figure 1: Geometry of tensile test samples a.) according to ISO 26203-2; b.) its modification as Generation I (Gen. I) sample and c.) further modification as Generation III (Gen. III) sample. EMA: elastic measurement area for strain gauge to measure the force; MinDA: minor deformation area; S1: first shoulder; S2: second shoulder, GL: gauge length of plastic deformation; d) FE-model of the test system. 
In this work, it was found, that the force oscillation is very large in the area of hydraulic jack and sliding bar for the sample loading, large in the area of load cell in the lower part of the test system, and nearly zero in the area of plastic deformation (GL area of the sample).

Based on these findings, many attempts were conducted to further reduce the force oscillations in the EMA between S1 and the second shoulder (S2) of the Gen. I specimen (Fig. 1b). In the new Gen. III sample, a cut-off between S2 and the sample clamping area was introduced [10]. This geometric section variation was selected at first to create a kind of geometric filter that may prevent the stress waves from being reflected back to the EMA when the downward stress waves are reflected at the lower end of the sample. With the decreasing cross-section in the weakening area in and below S2, a decreasing system ringing could be observed in the EMA. At the end, the best results were achieved when a slight plastic deformation occurs in the weakening area in S2, which is named the minor deformation area (MinDA), as shown in Fig. 1c. The effect of the slight plastic deformation is much larger than the pure geometric changes, i.e. the cross section reduction between EMA and the clamping area.

The conditions necessary to achieve these oscillation-free force measurements can be formulated using Fig. $2 \mathrm{a}$ where the Gen. III sample is divided in $\mathrm{n}=7$ sections. The test force is applied in section 1, while the specimen is firmly clamped and immovable in section 7 . In section 3 , the actual major plastic deformation up to fracture takes place. Section 5 is dimensioned larger than section 3. Next to section 5, another section 6 with geometric weaknesses is introduced. The dimensions of the specimen must fulfill different conditions:

Section 3:

$$
\frac{R_{m B 3} \cdot A_{B 3}}{R_{m B 6} \cdot A_{B 6}}<1
$$

This ensures that section 3 is mainly plastically deformed since the required deformation force here is lower than in section 6 . The sample deformations are measured to calculate the strains. For a homogeneous material, $R_{p 0.2}$ and $R_{m}$ are the same in all sections.

$\underline{\text { Section 5: }}$

$$
\frac{R_{m B 3} \cdot A_{B 3}}{R_{p 0.2 B 5} \cdot A_{B 5}} \leq 1
$$

This condition ensures that only pure elastic deformation may occur in section 5 .

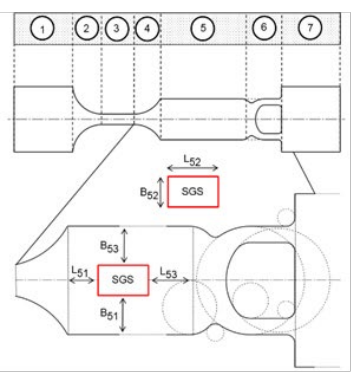

a)

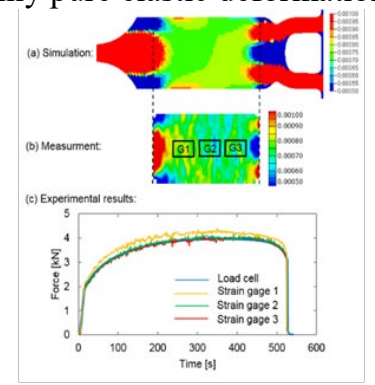

b)

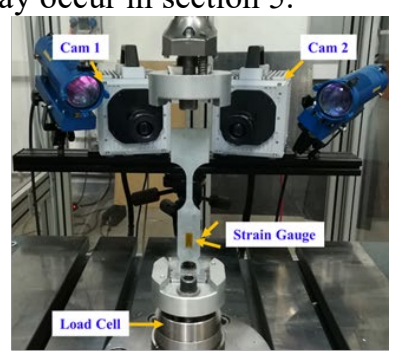

c)

Figure 2: a) detailed characteristic of Gen. III sample; b) FE-optimized section 5 and 6 of a) and forces measured by load cell and strain gages; c) test installation

$\underline{\text { Section } 6}$ (to ensure a slightly plastic deformation in this area while section 3 is majorly deformed):

$\frac{R_{p 0,2 B 6}}{R_{m B 3}} \leq \frac{A_{B 3}}{A_{B 6}}<\frac{R_{m B 6}}{R_{m B 3}}=1$ 
$\mathrm{A}_{\mathrm{B}(\mathrm{n})}$ : Cross-section in section (n) with (n) $=1-7 ; R_{p 0,2 B(n)}, \mathrm{R}_{\mathrm{mB}(\mathrm{n})}$ : yield and Tensile strength of the material in section (n)

In addition to these conditions, the shape and position of the local weakening in section 6 must be designed appropriately in accordance with section 5. A simple hole may reduce the force oscillations. However, the elastic stresses are inhomogeneous and thus the strain field is also inhomogeneous as shown in FEM [10]. The force measured in this area is then location dependent.

Using FEM a homogenous stress field was obtained (Fig. 2b). The quasi-static force measured by load cell is the same as the forces measured in position B and C of the Gen. III sample shown in Fig. 2b. Using the optimized geometry and the installation shown in Fig 2c, ringing free force-time (displacement) curves can be obtained without any data filtering (see Fig. 3a) on a DP 600 steel. The deformation of the sample was measured by using DIC (Company GOM, Aramis), carried out on images taken from two Photron SA5 high-speed cameras. The so converted true stress-strain curves are shown in Fig. 3b. Tests on different steel grades (DC04, HC340, DP1000, CPW1000) and Aluminium (Al 6082) show the same positive results.
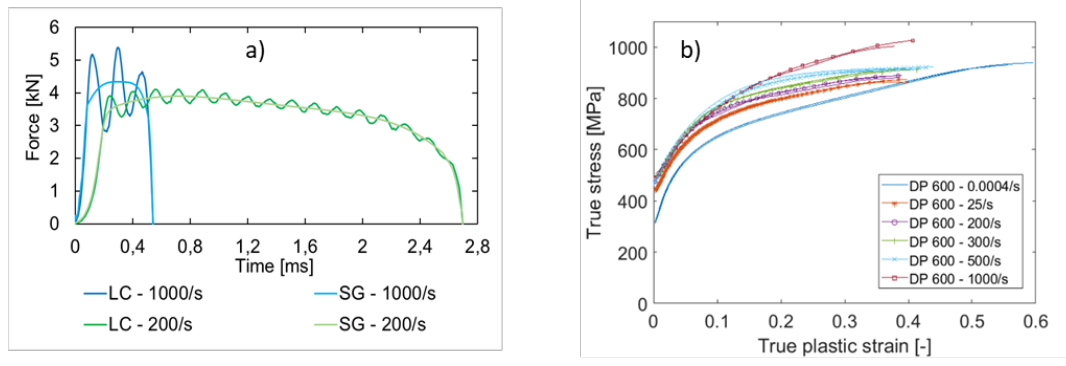

Figure 3: a) Forces measured using load cell and Strain gage (SG) on DP 600 steel (without any filter); b) the corresponding stress strain curves

\section{Model description}

To model and understand the physical background of the Gen. III sample, a simplified analytical one-dimensional stress wave model was developed [11]. In this model, the change of the geometric sections and the impedance $(\rho \cdot C)$ of the sample are considered, which determines the stress wave according to eqn. 1 . Here, $\rho$ is the material's density, and $\mathrm{C}$ represents the wave speed, which is determined by eqn. 5 in a general form [2]:

$$
C=\sqrt{\frac{1}{\rho_{0}} \cdot \frac{d \sigma}{d \varepsilon}}
$$

For elastic deformation, the hardening rate is equal to Young's modules E. In this case, the impedance can also be written as $\sqrt{E \cdot \rho_{0}}$. To use the elastic wave theory in this work, and to explain the Gen. III sample, the plastic deformation is considered as a non-linear elastic deformation [11]. For materials in the plastic deformation, the impedance must be rewritten as $\sqrt{d \sigma / d \varepsilon \cdot \rho_{0}}$. Here, the damping of plastic deformation is separated and the property $d \sigma / d \varepsilon$ is considered only.

The stress waves can be reflected and transmitted if they meet a change in the geometric section size or a change in the material properties (change in the hardening rate $d \sigma / d \varepsilon$, for example from a bar with an uniform cross section but two different materials: left side steel and right side $\mathrm{Al}$ ) or at the end of the bar or the specimen. Two waves (i.e., reflected and transmitted waves) also interact with each other if they meet in a certain section of the bar. 
Considering the case that a one-dimensional wave is reflected and transmitted on the crosssection or the material property changes, as shown in Fig. 4, the incident wave with wave stress $\sigma_{I}$ will be reflected, having a reflected wave stress $\sigma_{R}$ according to eqn. 6 and a transmitted wave according to eqn. 7 [2]:

$$
\begin{aligned}
& \sigma_{R}=F \cdot \sigma_{I} \\
& \sigma_{T}=T \cdot \sigma_{I} \cdot \frac{A_{1}}{A_{2}}
\end{aligned}
$$

with $\mathrm{n}, \mathrm{F}$ and $\mathrm{T}$ defined as follows:

$$
\begin{aligned}
& n=\frac{\left(\rho_{0} C_{0} A\right)_{1}}{\left(\rho_{0} C_{0} A\right)_{2}}=\frac{\left(\sqrt{\frac{d \sigma}{d \varepsilon} \rho} \cdot A\right)_{1}}{\left(\sqrt{\frac{d \sigma}{d \varepsilon} \rho} \cdot A\right)_{2}}=\frac{A_{1}}{A_{2}} \cdot \alpha \\
& F=\frac{1-n}{1+n} \\
& T=\frac{2}{1+n}
\end{aligned}
$$

with $\alpha$ as the ratio between Young's elastic modulus and the plastic modulus (work hardening rate), according to eqn. 11, if the waves are transmitting from a material section in the elastic state to a material section in the plastic deformation state. If both sections are in the elastic state, $\alpha=1$.
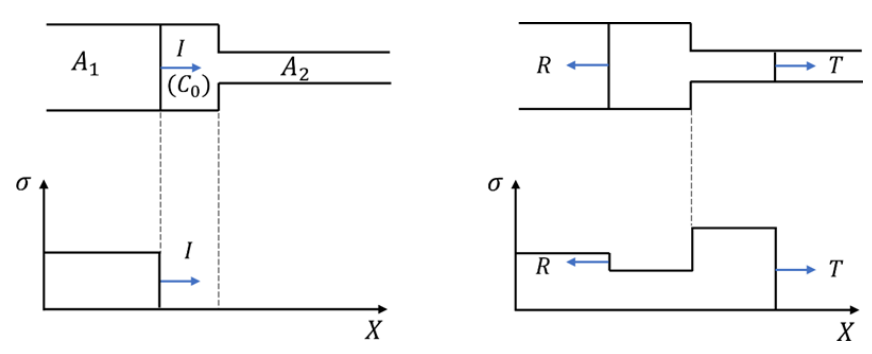

Figure 4: Reflection and transmission of stress waves in a bar with different cross sections

$$
\alpha=\sqrt{\frac{(d \sigma / d \varepsilon)_{e l}}{(d \sigma / d \varepsilon)_{p l}}}
$$

According to Wang [2] and Johnson [12], a stress wave with an infinitesimal short impulse length on a stepped bar with six in the wave direction decreasing section sizes will be transmitted and reflected on each section size changes. The stress waves in each section 
superimpose and increase from section to section in this case. This kind of stepped bar can work as a wave amplifier or attenuator.

Based on the concept of Wang and Johnson, The Gen. III sample is simplified by five straight one-dimensional beam sections 1-5 (Fig. 5a). Beam 1 represent the upper sample clamping area of the Gen III sample in Fig. 1c, beam 2 the major plastic deformation area or the GL area, beam 3 the EMA area for SG force measurement, beam 4 the MinDA with crosssection weakening where minor plastic deformation occurs, and finally beam 5 represents the lower sample clamping zone. The sections have different cross-sections (A1-A5), which are calculated by their width (w) multiplied by their thickness (d). They may have different elastic or elastic-plastic properties, which are described by their work hardening $d \sigma / d \varepsilon$ or Young's modulus E and density $\rho_{0}$.
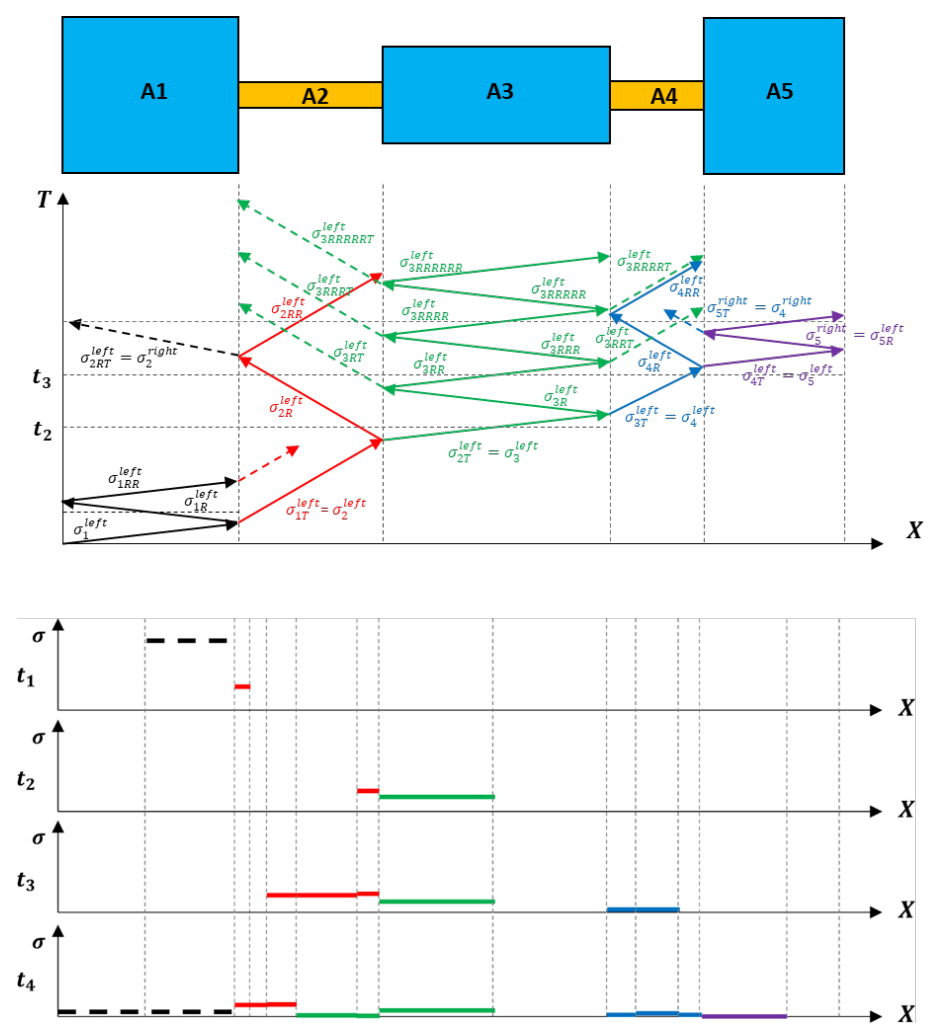

Figure 5: a.) Simplification of Gen. III sample to a one-dimensional beam model and the traces of the stress waves (reflections and transmissions); b.) the stress wave amplitude in different areas (constant $\alpha$-value of 8 was used), location $\mathrm{X}$ and time $\mathrm{t}, \mathrm{t} 1$ : begin of the wave propagation, $\mathrm{t} 2-\mathrm{t} 4$ : increasing time of wave propagation.

The impact stress pulse $\sigma_{I}=\sigma_{1}^{\text {left }}$ passes through beam 1 and is transmitted into beam section 2 at the boundary between the two beams $\left(\sigma_{1 T}^{\text {left }}\right)$, and reflected on the same $\left(\sigma_{1 R}^{\text {left }}\right)$. The transmitted and reflected stress can be calculated by eqn. $6-11$. The transmitted wave can propagate further to the boundary between beam 2 and 3 and so on. In this way, many stress waves are created as shown in Fig. 5a.

To obtain an approximated value of the stress wave amplitude in beam 3, where SG measurements show almost no ringing effect in Gen. III sample, several assumptions and simplifications were made [11]. Among them, it was assumed, that the second-order reflected 
waves, such as $\sigma_{1 R R}^{\text {left }}$ in beam 1 and the $\sigma_{2 R R}^{\text {left }}$ in beam 2, have not to be followed further. Here, it is assumed that the plastic deformation begins directly after the first wave transmission so that these two waves are absorbed by the plastic deformation in beam 2 and cannot be further transmitted into beam 3. This means that after $\sigma_{1}^{\text {left }}$ is transmitted into beam 3, it will be captured inside beam 3 and be reflected back and forth in Beam 3 after the plastic deformations occur in beams 2 and 4 . The $\sigma_{3 R \ldots R}^{\text {left }}$ is captured in beam 3 (see Fig. 5a), while on every boundary a part of it get lost due to the transmission (as $\sigma_{3 R \ldots R T}^{\text {left }}$ ) into beam 2 and 4 . As a consequence, the amplitude of $\sigma_{3 R \ldots R}^{\text {left }}$ decreases on every boundary.

The assumption of absorption of elastic stress wave by plastic deformation can be approved by several works and explained by metal physics [13-15]: if an elastic wave hits an individual dislocation, it will oscillate in response. The ensuing oscillatory motion will generate outgoing (from the dislocation position) elastic waves. When many dislocations are present, multiple scattering occurs and wave amplitude attenuates because of the energy scattered away from the direction of propagation. Detailed theoretical calculations have been performed [15] based on the Granato-Lücke theory.

\subsection{Constant work hardening and $\alpha$-value}

Considering the reflections of $\sigma_{3 R \ldots R}^{\text {left }}$ only, and using the geometric dimensions of Gen. III sample and the steel properties of a DP 600 steel $(\alpha=8=$ constant $)$, the stress wave amplitude changes can be seen in Fig. 5 b. With the increasing time, the stress wave amplitude decreases in Gen. III sample. For a DC04 mild steel, the $\alpha$-value is larger (ca.15 at the beginning of plastic deformation), the stress attenuation is even larger and faster.

For a constant $\alpha$-value, which means a constant work hardening rate $d \sigma / d \varepsilon$ over the entire plastic deformation range, the analytical one-dimensional model can be coupled with mathematical optimization, which can predict the sample geometry.

Using $A_{1}=A_{5}=40 \mathrm{~mm}^{2}$ and $A_{2}=6.5 \mathrm{~mm}^{2}, A_{3}=25 \mathrm{~mm}^{2}, A_{4}=7.5 \mathrm{~mm}^{2}$, which is the geometry of Gen. III sample in Fig. 1c, and the $\alpha=8$ (value of the beginning of the plastic deformation of a DP 600 steel), it can be calculated that $\mathrm{N}=18$ reflections inside of beam 3 are needed to attenuate the stress wave amplitude of incident stress $\sigma_{I}$ to $1 \%$ of its initial value. Using beam 3's length of $50 \mathrm{~mm}(\mathrm{EMA}=50 \mathrm{~mm}$; see Fig. 1c), this takes at a steel sound speed of $5800 \mathrm{~m} / \mathrm{s} 0.15 \mathrm{~ms}$. When larger $\alpha$-values are used, which means a smaller work hardening rate, the $\mathrm{N}$-value decreases and can be even $\mathrm{N}=1$. This means that the fluctuation of forces measured in EMA of Fig. 1c may depend on the work hardening rate, and thus are material-dependent.

This phenomenon has been overlooked [10] for DP 600 (Fig. 3) with a relatively small $\alpha$ value at the beginning of the deformation because the force-time-curves are the average of 3 to 5 tests and the small force oscillations are smoothed.

When looking at single force-time curves shown in Fig. 6, a small force oscillation may be recognized for DP 600 steel up to $0,2 \mathrm{~ms}$ after the beginning of the plastic deformation. This oscillation decreases with the time. The ferritic stainless steel FSS439, which has a much smaller work hardening rate comparing to DP 600 (which means a larger $\alpha$-value), shows very little initial force oscillation. For DC04 steel with very small work hardening rate and thus larger $\alpha$-value, no force oscillation can be observed. The prediction of analytical model is thus approved.

Starting with a fixed $\alpha$-value of $\alpha=10$, which is in between DP 600 and DC04, if in the optimization the conditions are set as follows: A1 $=\mathrm{A} 5=40 \mathrm{~mm}^{2}, \mathrm{~A} 2=6.5 \mathrm{~mm}^{2}, 10 \mathrm{~mm}^{2}<$ A $3<60 \mathrm{~mm}^{2}$, and $5.5 \mathrm{~mm}^{2}<\mathrm{A} 4<10 \mathrm{~mm}^{2}$, then the optimization results using the software developed in [11] are: $\mathrm{A} 3=12.5 \mathrm{~mm}^{2}$, and $\mathrm{A} 4=6.6 \mathrm{~mm}^{2}$, if the stress amplitude in beam 3 
should be only $2 \%$ of the initial incident stress value. For $5 \%$, A3 $=47 \mathrm{~mm}^{2}$, and A4 $=7.9$ $\mathrm{mm}^{2}$ were obtained. In addition, for any reasonable values of the parameters, A4 is always larger than A2. This confirms that beam 4 must be stronger than beam 2, as found in previous studies [10] and as Gen. III in Fig. 1c shows.

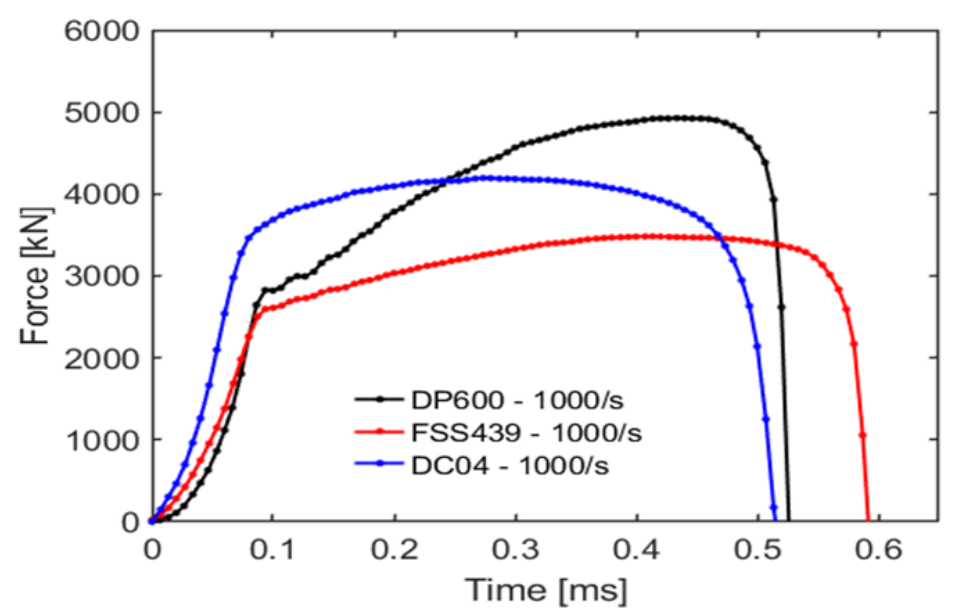

Figure 6: Force-time curve measured in EMA area of Gen. III sample for three steels

\subsection{Variable work hardening and $\alpha$-value}

Section 3.1 uses constant $\alpha$-value, which is a simplification. The general case is that $\alpha$ value is increasing with the time, since the work hardening rate of the metals generally decreases with the plastic deformation. In this case, every single wave in the sample shown in Fig. 5a must be calculated and followed.

Using the equations in [11] and the geometric dimensions of Gen. III sample: $\mathrm{A} 1=\mathrm{A} 5$ $=40 \mathrm{~mm}^{2}, \mathrm{~A} 2=6.5 \mathrm{~mm}^{2}, \mathrm{~A} 3=25 \mathrm{~mm}^{2}, \mathrm{~A} 4=7.5 \mathrm{~mm}^{2}$ and $\mathrm{L} 1=45 \mathrm{~mm}, \mathrm{~L} 2=37 \mathrm{~mm}, \mathrm{~L} 3=$ $58 \mathrm{~mm}, \mathrm{p}=0.02$, and the variable $\alpha$-values shown in Fig. 7, the reduction of the stress wave amplitude (normalized to the initial incident stress amplitude $\sigma_{1}^{\text {left }}$ ) can be seen in Fig. 7 for both DC04 and DP 600 steels.

It is obvious that the reduction of stress wave amplitude is strongly dependent on the value and shape of the $\alpha$-value. For the original Gen. III sample geometry, more time are needed for DP 600 steel to attenuate the stress amplitude in comparison to DC04. Therefore, DP 600 may show some force oscillations at the beginning of the test, and it will disappear with the time. In addition, due to the wave travel time $\alpha_{4}$ is always smaller than $\alpha_{2}$. Thus, the plastic deformation in beam 4 is always slightly delayed in comparison to beam 2 . The findings with constant $\alpha$-value are thus confirmed also here with the more realistic variable $\alpha$-value.

Theoretically, the geometry of the Gen. III sample must be slightly adjusted for each material due to their differences in work hardening rate and thus $\alpha$-value. However, in practice, since the stress strain curves are averaged on several tests, the small oscillations are smoothened as shown in Fig. 3. The geometry in Fig. 1c can thus be used as a standard. 


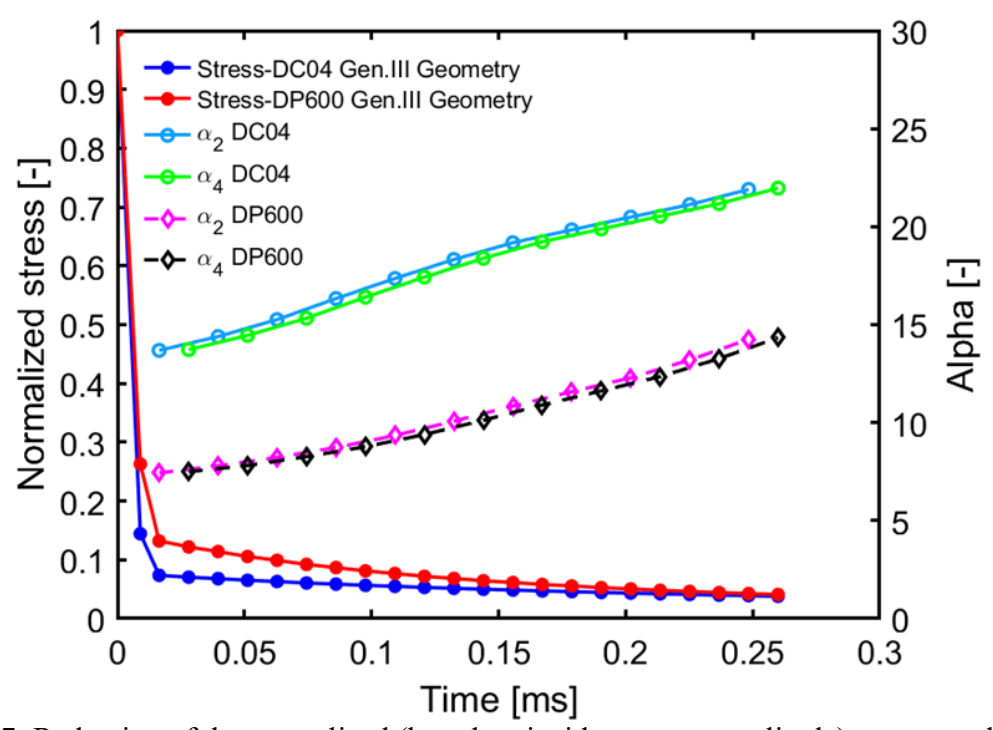

Figure 7: Reduction of the normalized (based on incident stress amplitude) stress amplitude during the tests and the corresponding $\alpha$-value in beam 2 and 4 for DC04 and DP 600 steels

\section{Summary and conclusion}

The entire servo-hydraulic high-speed test system of company zwick (HTM 5020) was modelled using LS-Dyna. Based on the findings of stress wave amplitude differences in different area of the system, and the observation that the stress wave amplitude is zero in plastic deformation area, a novel Gen III specimen geometry with a corresponding measuring method was developed in this work. By introducing a new minor plastic deformation area with specials geometric features, an oscillation-free force measurement at strain rates of up to $1000 / \mathrm{s}$ was realized.

The geometry of the sample can be approximately predicted by a one-dimensional stress wave model based on several simplifications and assumption. The model may explain the physical background of new sample well since it predicts the sample geometry qualitatively correct, and it may predict one phenomenon, which has been overseen up to now.

This new sample can be used for the entire strain rate range of $10^{-4}-10^{3} / \mathrm{s}$. The problems of using different samples and measuring techniques for different strain rate ranges are solved, and artificial effects are avoided.

This work was supported by the German Research Foundation (Deutsche Forschungsgemeinschaft, Grant No. FA 1024/5-1), to which I am indebted.

\section{References}

1. T.J. Cloetea, M. Stander: Refinement of the wedge bar technique for compression tests at intermediate strain rates, Proceeding EPJ Web of Conferences 26, 01025, DYMAT 2012

2. L.L. Wang: Foundations of Stress Waves, Elsevier, 2007

3. X. Xiao: Dynamic tensile testing of plastic materials, Polymer Testing 27, 164-178 (2008) 
4. S. Hill, M. Pinnell, A. Minch: Standardization of High Strain Rate Tensile Testing of Polymers, paper presentation, ANTEC, Boston, 2005

5. B. Yan, Y. Kuriyama, A. Uenishi, D. Cornette, M. Borsutzki, C. Wong: Recommended Practice for Dynamic Testing for Sheet Steels-Development and Round Robin Tests. SAE Transactions 155, 147-157 (2006)

6. D. Bruce, D. Matlock, J. Spee, A. De: Assesment of strain-rate dependent tensile properties of automotive sheet steels. SAE Technical paper 2004-01-0507

7. W. Böhme: FAT-Richtlinie Dynamische Werkstoffkennwerte für die Crashsimulation. Mater Test 50, 199-205 (2008), https://doi.org/10.3139/120.100865

8. P. Verleysen: Numerical study of the influence of the specimen geometry on split Hopkinson bar tensile test results, Latin American Journal of Solids and Structures 6, 285-298 (2009)

9. J. Li and X.F. Fang: Stress Wave Analysis and Optical Force Measurement of ServoHydraulic Machine for High Strain Rate Testing, Exp Mech. 54, 1497-1501 (2014)

10. X.F. Fang and R. Grams: A Novel Oscillation-Free Force Measurement for the Determination of Material Properties during High-Speed Tests, ASTM Journal of Testing and Evaluation, (2019), doi:10.1520/JTE20180887

11. X. F. Fang, ,A one-dimensional stress wave model for analytical design and optimization of oscillation-free force measurement in high-speed tensile test specimens", International Journal of Impact Engineering, 149, 103770 (2021), https://doi.org/10.1016/j.ijimpeng.2020.103770

12. W. Johnson, Impact Strength of Materials, Edward Arnold, London, 1972

13. A. Maurel, J-F. Mercier and F. Lund, Elastic wave propagation through a random array of dislocations, PHYSICAL REVIEW B 70, 024303 (2004), DOI: 10.1103/PhysRevB.70.024303

14. S. Tasdemirci and I.W. Hall, The effects of plastic deformation on stress wave propagation in multi-layer materials, Int J Impact Eng, 34, 1797-1813 (2007)

15. A. Maurel, V. Pagneux, F. Barra and F. Lund, Wave propagation through a random array of pinned dislocations: Velocity change and attenuation in a generalized Granato and Lücke theory. PHYSICAL REVIEW B 72, 17411 (2005) DOI:

10.1103/PhysRevB.72.174111 